\title{
Preemptive Scheduling of Uniform Machines Subject to Release Date
}

\author{
J. Labetoulle \\ Centre National d'Etudes des Telecommunications \\ Issy les Moulineaux, France \\ E.L. Lawler \\ University of California \\ Berkeley, U.S.A. \\ J.K. Lenstra \\ Centre for Mathematics and Computer Science \\ Amsterdam, The Netherlands \\ A.H.G. Rinnooy Kan \\ Erasmus University \\ Rotterdam, The Netherlands
}

We shall be concerned with finding optimal preemptive schectules on paralle machines, subject to release dates for the jobs. Two polynomial-time algorithms are presented. The first algorithm minimizes maximum completion time on an arbitrary number of uniform machines. The second algorithrn minimizes maximum lateness with respect to due dates for the jobs on an arbitrary number of identical machines or on two uniform machines. A third algorithm for minimizing maximum lateness on an arbitrary number of uniform machines is briefly discussed. NP-hartness is established for the problem of minimizing rotal weighted completion time on a single machine.

\section{Introduction}

We consider scheduling problems in which $n$ independent jobs $J_{1} \ldots \ldots J_{n}$ have to be processed on $m$ parallel machines $M_{1}, \ldots, M_{\text {m. }}$. Each machine can handle at most one job at a time and each job can be executed on at most one machine at a time. Each job

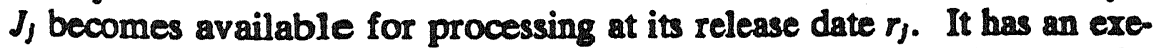
cution requirement $\boldsymbol{p}_{j}$ and possibly also a due date or deadline $d_{j}$ and a 
weight $w_{j}$. Unlimited preemption is allowed: the processing of any job may arbitrarily often be interrupted and resumed at the same time on a different machine or at later time on any machine. The machines are assumed to be uniform, i.e., each machine $M_{i}$ has a speed $s_{i}$, and complete execution of $J_{j}$ on $M_{i}$ would require $p_{j} / s_{i}$ time units. If all speeds are equal, the machines are identical; if $m=1$, we have a single machine. We assume that all numerical data $r_{j}, p_{j}, d_{j}, w_{j}, s_{l}$ are integers.

A feasible schedule defines a completion time $C_{j}$ and a lateness $L_{j}=C_{j}-d_{j}$ for each $J_{j}$. We may choose to minimize the maximum completion time $C_{\max }=\max _{1 \leq j \leq m}\left\{C_{j}\right\}$, the maximum lateness $L_{\max }=\max _{1 \leq j \leq n}\left\{L_{j}\right\}$, the total completion time $\sum C_{j}=\sum_{j=1}^{n} C_{j}$, or the total weighted completion time $\sum w_{j} C_{j}=\sum_{j=1}^{n} w_{j} C_{j}$.

When scheduling jobs subject to release dates, one can distinguish between three types of algorithms. An algorithm is on-line if at any time only information about the available jobs is required. It is nearly on-line if in addition the next release date has to be known. It is offline if all information is available in advance.

In Section 2 we consider the minimization of $C_{\max }$ on $m$ uniform machines. For the case that all release dates are equal, Horvath, Lam and Sethi [8] derived a closed form expression for the optimum value of $C_{\text {max. }}$. Gonzalez and Sahni [6] proposed an $O(m \log m+n)$ algorithm which produces a schedule meeting this value and containing at most $2(m-1)$ preemptions. For the case that the release dates are arbitrary, Sahni and Cho [18] gave an $O(n \log n+m n)$ off-line algorithm to determine if there exists a schedule in which no job is completed after a common deadline. We will present an $O\left(n^{2}\right)$ nearly on-line algorithm to minimize $C_{\max }$; Sahni and Cho [17] independently developed an $O\left(m n \log n+m^{2} n\right)$ nearly on-line algorithm that is very similar to ours. We will indicate how to obtain an $O(n \log n+m n)$ off-line implementation of our algorithm. These methods can also be used to minimize $L_{\operatorname{maz}}$ in the case of equal release dates.

In Section 3 we consider the minimization of $\mathbb{L}_{\max }$. For the case of equal release dates, Horn [7] proposed an $O\left(n^{2}\right)$ algorithm to minimize $L_{\max }$ on $m$ identical machines. For the case of arbitrary release dates, he gave an off-line algorithm, based on a network flow computation, to determine if there exists a schedule in which no job is completed after its deadline. Bruno and Gonzalez [3] adapted this feasibility test to the case of two uniform machines. We will extend both methods by presenting polynomial-time algorithms to minimize $L_{\text {max }}$. Martel $[13,14]$ recently proposed a feasibility test for the case of $m$ uniform machines, based on a polymatroidal network flow model, 
and used it to obtain a polynomial-time algorithm to minimize $L_{\operatorname{mas}}$. We will discuss these results as well.

In Section 4 we consider the minimization of $\sum C_{j}$ and $\sum w_{j} C_{j}$. For the case of equal release dates, Bruno and Gonzalez [5] proposed an $O(n \log n+m n)$ algorithm to minimize $\sum C_{j}$ on $m$ uniform machines. It is well known that in the case of identical machines allowing preemptions will not decrease the optimal value of $\sum w_{j} C_{j}[15]$. It follows that $\sum w_{j} C_{j}$ is minimized on a single machine by scheduling the jobs in order of nonincreasing ratios $w_{j} / p_{j}[19]$, and that the problem on two identical machines is already NP-hard $[2,12]$. For the case of arbitrary release dates, $\Sigma C_{j}$ is minimized on a single machine by an obvious on-line extension of the above ordering rule [1]; we will establish $N P$-hardness for the problem of minimizing $\sum w_{j} C_{j}$.

In Section 5 we conclude by indicating a major open problem and some important recent developments in the area of preemptive scheduling.

\section{Maximum Completion Time}

We first consider the problem of minimizing the maximum completion time $C_{\max }$ on $m$ uniform machines. The jobs and the machines are assumed to be ordered in such a way that $r_{1} \leq \cdots \leq r_{n}$ and $s_{1} \geq \cdots \geq s_{\text {m. }}$.

We will describe a nearly on-line algorithm that considers the time intervals $R_{k}=\left[r_{k}, r_{k+1}\right]$ in order of increasing $k$. For each successive interval $R_{k}(k=1, \ldots, n-1)$, denote the remaining execution requirement of $J_{j}$ at $r_{k}$ by $p^{(k)}(j=1, \ldots, k)$ and renumber the jobs so that $p_{1}^{(k)} \geq \cdots \geq p^{(k)}$. The subalgorithm to be applied in each interval determines the amounts by which the $p k^{(k)}$ are to be decreased within $R_{k}$. At time $r_{n}$, all jobs are available, and it is well known [8] that the minimum time for their completion is given by

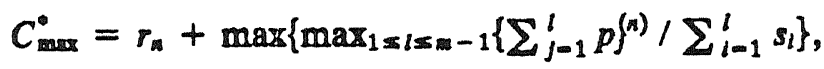

$$
\begin{aligned}
& \left.\left.\sum_{j=1}^{n} p\right\}^{(n)} / \sum_{l=1}^{m} s_{i}\right\} \text {. }
\end{aligned}
$$

The portion of an optimal schedule within any interval $R_{k}$ can be constructed by applying the Gonzalez-Sahni algorithm [6] to the quantities $p f^{(k)}-p f^{(k+1)}$ determined by our subalgorithm. Similarly, a schedule for the final interval $\left[r_{n}, C_{\max }^{*}\right]$ can be constructed by applying the same algorithm to the quantities $p^{(n)}$. Since both the subalgorithm and the schedule construction procedure require $O(n)$ time for each interval, the algorithm requires $O\left(n^{2}\right)$ time overall; it introduces $O(m n)$ preemptions into the optimal schedule. 
Our algorithm has the property that the remaining execution requirements passed on to the nert interval will be as evenly distributed as possible. More specifically, for each $k$ there is no way to process the jobs before $r_{k}$ that could lead to a smaller value for any of the partial sums $\sum_{j=1}^{l} p^{(k)}(l=1, \ldots, k)$. This immediately implies the correctness of the algorithm, since each of these partial sums appearing in (1) is as small as it could possibly be.

Rather than giving an inductive proof of this property, we will settle for a simpler correctness proof of the entire algorithm. This proof will also serve to introduce algorithmic refinements, by which the optimum value $C_{m}^{\circ}$ can be determined in $O(n \log n+m n)$ time. An actual schedule can be constructed by applying the Sahni-Cho algorithm [18], using $C_{\text {as a }}^{\circ}$ asmmon deadline for the jobs. This off-line approach requires $O(n \log n+m n)$ time and introduces $O(m n)$ preemptions into the optimal schedule.

Let us consider an interval $\boldsymbol{R}_{\boldsymbol{k}}$ for fixed $k$. Given the $p{ }^{(k)}(j=1, \ldots, k)$, we have to determine the $p f^{(k+1)}$ to be passed on to the nert interval $\boldsymbol{R}_{k+1}$.

Suppose that at time $r_{k}$ the jobs $J_{1}, \ldots, J_{v}$ are available and not yet completed, with $p_{1}^{(k)} \geq \cdots \geq p_{v}^{(k)}>0$. For ease of notation, we drop the superscripts. Thus, denote the given $p^{(k)}$ by $p_{j}$ and the unknown $p\}^{(k+1)}$ by $q_{j}(j=1, \ldots, v)$, and let $t=r_{k+1}-r_{k}$. For purposes of exposition, we assume for the time being that, if $m<v$, machines $M_{m+1}, \ldots, M_{v}$ with $s_{m+1}=\cdots=s_{v}=0$ are added to the model.

The $p_{j}$ can be viewed as defining a staircase pattern as in Figure 1. The $q$, will be chosen in such a way that they define a similar pattern.

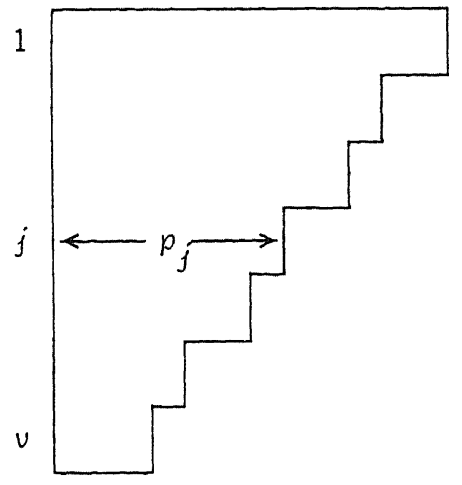

Figure 1. Staircase pattern at $r_{\mathrm{k}}$

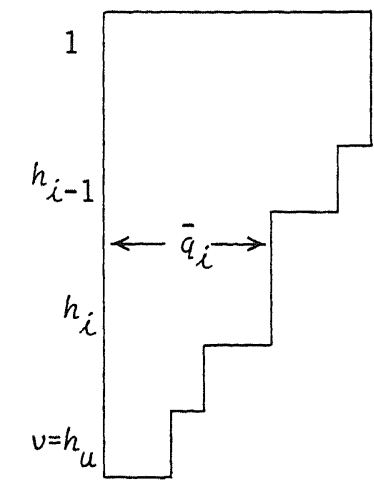

Figure 2. Staircase pattern at $r_{k+1}$.

As illustrated in Figure 2, such a staircase can be characterized by a 
sequence $\left(\left(h_{1}, \bar{q}_{1}\right), \ldots,\left(h_{w}, \bar{q}_{s}\right)\right)$, where $\bar{q}_{i}=q_{j}$ for each $J_{j}$ with $h_{i-1}+1 \leq j \leq h_{i}\left(i=1, \ldots, u ; h_{0}=0 ; h_{m}=v\right)$. A first condition for feasibility is that

$$
\bar{q}_{i}>\bar{q}_{l+1}(i=1, \ldots, k-1) .
$$

The staircase $\left(\left(h_{1}, \bar{q}_{1}\right), \ldots,\left(h_{m}, \bar{q}_{w}\right)\right)$ will be constructed in such a way that, for $i=1, \ldots, u-1$, the capacity of $M_{h_{i-1}+1} \ldots, M_{h_{l}}$ will be fully utilized to decrease $p_{h_{l-1}+1} \ldots \ldots p_{h_{l}}$ to $\bar{q}_{l}$. A second condition for feasibility is therefore that

$$
\begin{aligned}
\sum_{j=h_{l-1}+1}^{l} q_{j}=\left(l-h_{l-1}\right) \bar{q}_{l} & \geq \sum_{j=h_{l-1}+1}^{l} p_{j}-t \sum_{j=h_{l-1}+1}^{l} s_{j} \\
(l & \left.=h_{l-1}+1, \ldots, h_{l} ; i=1, \ldots, u\right),
\end{aligned}
$$

with the corners of the staircase, except possibly the last one, corresponding to strict equalities:

$$
\begin{gathered}
\sum_{j=h_{l-1}+1}^{h_{l}} q_{j}=\left(h_{l}-h_{l-1}\right) \bar{q}_{t}=\sum_{j=h_{l-1}+1}^{h_{i}} p_{j}-t \sum_{j=h_{l-1}+1}^{h_{i}} s_{j} \\
(i=1, \ldots, u-1) .
\end{gathered}
$$

A third condition for feasibility is of course that

$$
0 \leq q_{J} \leq p_{j}(j=1, \ldots, v) .
$$

We tentatively construct the first step of the staircase by setting

$$
h_{1}=1, \bar{q}_{1}=p_{1}-t s_{1} \text {. }
$$

Generally, having found $i$ tentative steps $\left(h_{1}, \bar{q}_{1}\right), \ldots,\left(h_{i}, \bar{q}_{i}\right)$ with $h_{i}<v$ and $\bar{q}_{1}>\cdots>\bar{q}_{i}$, we construct the $(i+1)$-st tentative step by setting

$$
h_{l+1}=h_{l}+1, \bar{q}_{l+1}=p_{h_{l+1}}-t s_{h_{l+1}} .
$$

If $\bar{q}_{l}>\bar{q}_{i+1}$ and $\bar{q}_{i} \geq 0$, the staircase $\left(\left(h_{1}, \bar{q}_{1}\right), \ldots,\left(h_{1+1}, \bar{q}_{l+1}\right)\right)$ satisfies (2) and (4); we increment $i$ by one and, if $h_{i}$ is still smaller than $v$, construct the nert step.

Suppose now that $\bar{q}_{i} \leq \bar{q}_{t+1}$ or $\bar{q}_{l}<0$. In the latter situation, there is excess capacity on $M_{h_{l-1}+1}, \ldots, M_{h_{i}}$; in both cases, some of the capacity of these machines has to be devoted to processing $J_{h_{l}+1}$ if (2) and (4) are to be satisfied. We therefore reconstruct the $i$-th step so as to include $J_{h_{l}+1}$ as well: $h_{l}$ is incremented by one, and $\bar{q}_{t}$ is recalculated according to

$$
\bar{q}_{i}=\left(\sum_{j=h_{l-1}+1}^{h_{l}} p_{j}-t \sum_{j=h_{l-1}+1}^{h_{l}} s_{j}\right) /\left(h_{l}-h_{l-1}\right)
$$


(cf. (4)). As a result, it may now be that $\bar{q}_{i-1} \leq \bar{q}_{i}\left(\bar{q}_{i-1}<0\right.$ cannot occur). In this case, we reconstruct the $(i-1)$-st step so as to include the current $i$-th step: $h_{i-1}$ is increased to $h_{i}$, and $\bar{q}_{t-1}$ is recalculated as in (7). We continue until once more $\bar{q}_{1}>\ldots>\bar{q}_{i}$; the adjusted staircase $\left(\left(h_{1}, \bar{q}_{1}\right), \ldots,\left(h_{i}, \bar{q}_{i}\right)\right)$ includes one more job and may have fewer steps than before. If $h_{i}$ is still smaller than $v$, we construct the nest step according to (6).

The process is terminated as soon as $h_{i}=v$. If $\bar{q}_{i}<0$, we reset $\bar{q}_{i}=0$ and note that only in this situation the last corner of the staircase does not correspond to a strict equality.

We have to verify that the resulting staircase $\left(\left(h_{1}, \bar{q}_{1}\right), \ldots,\left(h_{u}, \bar{q}_{u}\right)\right)$ and the corresponding remaining execution requirements $q_{1}, \ldots, q_{v}$ indeed satisfy the feasibility conditions (2) (5). For (2) and (4), this is obvious. To see that (3) must be true, note that each $\bar{q}_{i}$ is initially defined by an equality constraint and can only increase thereafter. To verify (5), it is sufficient to show that $\bar{q}_{i} \leq p_{h_{i}}$. Subtracting (3) for $l=h_{i}-1$ from (4), we find $\bar{q}_{t} \leq p_{h_{l}}-t s_{h_{l}}$, which implies the desired result.

Let us now analyze the running time of the subalgorithm. The number of step constructions as in (6) is exactly $v$. The number of step reconstructions as in (7) is at most $v-1$, since during each adjustment two steps are collapsed into one. It follows that the process terminates in $O(v)$ time. This presupposes that the given values $p_{j}$ are ordered; but since the relative order of the remaining execution requirements does not change, we can maintain an ordered list of these values and insert the value of the job that becomes available at $r_{k}$ in $O(v)$ time. Hence the subalgorithm determines the values $q$, for each interval in $O(v)$ time. As has been indicated above, the Gonzalez-Sahni algorithm [6] can be applied to construct an actual schedule in each interval in $O(v)$ time as well. We thus have arrived at a nearly on-line algorithm that requires $O\left(n^{2}\right)$ time overall.

We now intend to prove the correctness of the algorithm.

We note first that not only does the relative order of the remaining execution requirements remain invariant, but also the following stronger property holds: as soon as two remaining execution requirements become equal, they will remain equal. To see this, suppose that $p_{j}=p_{j+1}$ at time $r_{k}$, and let $h_{i}=j$. According to (6), we set $\bar{q}_{l+1}=p_{j+1}-t s_{j+1}$. But $\bar{q}_{i} \leq p_{j}-t s_{j} \leq p_{j}-t s_{j+1}=\bar{q}_{l+1}$, and we have to reconstruct the $i$-th step so as to include $J_{j+1}$ as well.

This leads us to define the rank of an available job $J_{j}$ at time $r_{k}$ as the value $h_{l}$ for which $h_{i-1}+1 \leq j \leq h_{l}$. The rank of a job at time $r_{n}$ is defined analogously as its step height that would be found if the 
subalgorithm were to be applied in the interval $\left[r_{n}, C_{\max }^{\circ}\right]$. A job will be called critical if its rank is at most $m-1$ and moncritical otherwise. The rank of a job cannot decrease; in particular, once a job becomes noncritical, it never becomes critical again. It follows from (4) that in any interval the fastest $h_{i}$ machines are exclusively processing the longest $h_{i}$ critical jobs. A critical job is processed continuously from its release date until it either is completed or becomes noncritical.

These observations suggest the following correctness proof for the algorithm. First, suppose that the schedule ends at $C_{\max }^{*}$ with the simultaneous completion of $l$ critical jobs $(l<m)$. At any time when $l^{\prime}$ of these jobs are available, they are processed by the fastest $l^{\prime}$ machines. In this case, the schedule is clearly optimal.

Alternatively, suppose that the schedule ends with the simultaneous completion of $m$ noncritical jobs. Let $r_{k}$ be the last release date just prior to which there is idle time on some machine. Ignoring the jobs that are available but noncritical at time $r_{k-1}$, we conclude that the portion of the schedule for the remaining jobs has a structure as illustrated in Figure 3.

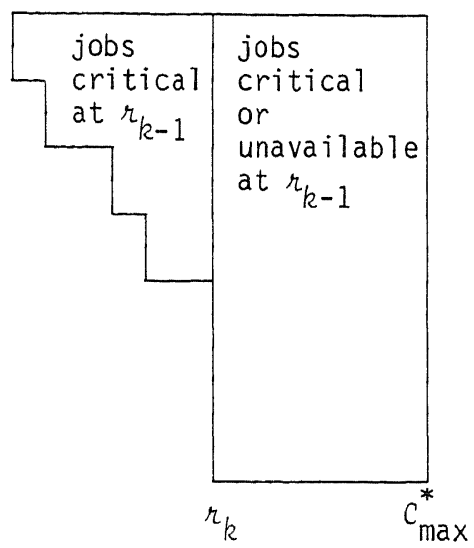

Figure 3 - Simultaneous completion of noncritical jobs.

Before $r_{k}$, the available critical jobs are processed by the fastest machines. Between $r_{k}$ and $C_{\max }^{\circ}$, there is no idle time. It follows that the schedule is optimal for the jobs under consideration and a fortiori that $C^{\circ}$ is the minimum time to complete all the jobs.

Let us use the new terminology to describe a more efficient implementation of the subalgorithm. We will reduce the running time by dealing more carefully with the noncritical jobs, circumventing the need to introduce machines of speed zero.

Consider the situation after a typical application of the 
subalgorithm, as illustrated in Figure 4.

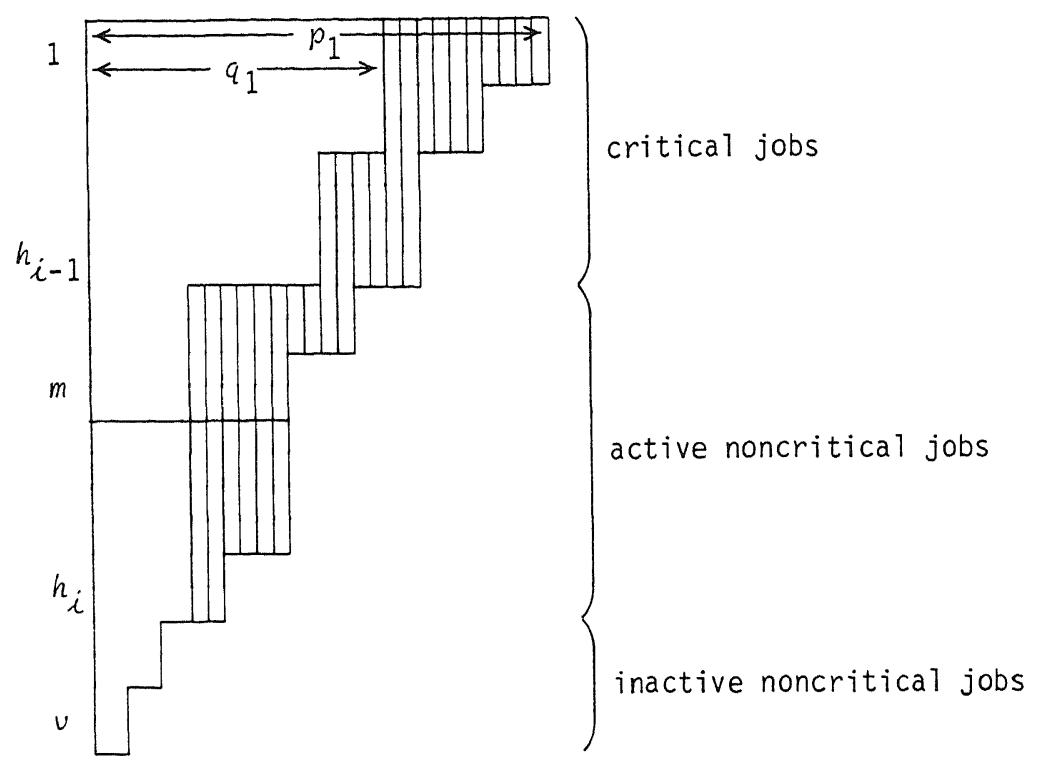

Figure 4 - Staircase patterns at $r_{k}$ and $r_{k+1}$.

The noncritical jobs of lowest rank, i.e., $J_{h_{i-1}+1}, \ldots, J_{h_{i}}$ where $h_{t-1}+1 \leq, m \leq h_{i}$, will be called active. In the interval $R_{k}$, their remaining execution requirements are reduced by machines $M_{h_{l-1}+1}, \ldots, M_{m}$ to a common amount $\bar{q}_{l}$. The remaining noncritical jobs, i.e., $J_{h_{i}+1}, \ldots, J_{v}$, will be called inactive. In $R_{k}$, their remaining execution requirements are not reduced at all, since $\bar{q}_{i}>\bar{q}_{i+1}=p_{h_{i+1}}$ (note that $s_{\mathrm{h}_{i+1}}=0$ ).

As a first refinement, the subalgorithm does not have to deal with the active noncritical jobs separately, since their remaining ezecution requirements will remain equal throughout. They can easily be handled simultaneously by straightforward generalizations of (6) and (7). As a second refinement, the subaigorithm can be terminated as soon as either $h_{b}=v$ or $h_{i} \geq m$ and $\bar{q}_{l}>p_{h_{l}+1}$.

Rather than maintaining an ordered list of all remaining execution requirements, we have to do so only for the largest $m-1$ of them. We simply record the number of active noncritical jobs, their common remaining execution requirement, and the lowest inder of any of them. Finally, we maintain a priority queue for the remaining requirements of the inactive noncritical jobs.

At each release date, the execution requirement of the job that 
becomes available is, depending on its size, inserted either in the ordered list in $O(m)$ time or in the priority queue in $O(\log n)$ time. The staircase computations for the longest $m-1$ jobs and the active noncritical jobs require $O(m)$ time in each interval and $O(\mathrm{mn})$ time overall. The queue operations require $O(\log n)$ time in each interval and $O(n \log n)$ time overall, since once an inactive job becomes active and is withdrawn from the queue, it remains active throughout. Hence successive applications of the modified subalgorithm determine the value $C_{\operatorname{mox}}^{\circ} \mathrm{O}(n \log n+m n)$ time. As has been indicated above, the Sahni-Cho algorithm [18] can be applied to construct an actual schedule in the interval $\left[r_{1}, C_{\max }^{6}\right]$ in $O(n \log n+m n)$ time as well. We thus have arrived at an off-line algorithm that requires $O(n \log n+m n)$ time overall.

\section{Maximum Lateness}

We now consider the problem of minimizing the maximum lateness $L_{\max }$ on $m$ identical machines.

A relaxed version of this problem is to test a trial value of $L_{\max }$ for feasibility. That is, for a given value $y$, one has to determine whether or not there exists a schedule for which $L_{\max } \leq y$. This condition is equivalent to the requirement that no job $J_{j}$ is completed after an induced deadline $d_{j}+y$. Sahni [16] proposed an off-line algorithm for the case of equal deadlines that requires $O(n \log m n)$ time and introduces at most $n-2$ preemptions. He also showed that there can be no nearly on-line algorithm for the case of arbitrary deadlines. Horn [7] proposed a network flow algorithm for the latter case. He suggested that one might conduct a search for the optimum value of $\boldsymbol{L}_{\max }$, but offered no upper bound on the number of trial values that have to be tested. Our contribution here is to obtain such a bound and to show that it is polynomial in the problem size.

Horn's approach is as follows. Suppose $y$ is a trial value for $L_{\max }$. Let $\left\{e_{1}, \ldots, e_{2 n}\right\}\left(e_{1} \leq \ldots \leq e_{2 n}\right)$ be the ordered collection of release dates $r_{j}$ and induced deadlines $d_{j}+y$; if a release date and a deadline are equal, the smaller inder is to be assigned to the release date. Further, define the time interval $E_{k}=\left[e_{k}, e_{k+1}\right]$ for $k=1, \ldots, 2 n-1$.

A flow network is constructed with job vertices $J_{1}, \ldots, J_{n}$, interval vertices $E_{1}, \ldots, E_{2 n-1}$, a source vertex $S$ and a sink vertex $T$. There is an $\operatorname{arc}\left(J, E_{k}\right)$ of capacity $e_{k+1}-e_{k}$ if and only if $r_{j} \leq e_{k}$ and $e_{k+1} \leq d_{j}+y$. In addition, there is an arc $\left(S, J_{j}\right)$ of capacity $p_{j}$ for $j=1, \ldots, n$ and an arc $\left(E_{k}, T\right)$ of capacity $m\left(e_{k+1}-e_{k}\right)$ for $k=1, \ldots, 2 n-1$. Now, a maximum value flow is found in $O\left(n^{3}\right)$ time 
$\left\{J_{j} \mid j \in X\right\}$ and each vertex $E_{k}$, it is required that the total flow through the set of $\operatorname{arcs}\left\{\left(J_{j}, E_{k}\right) \mid j \in X\right\}$ is no more than $p(k,|X|)$, where

$$
p(k, l)=\left(e_{k+1}-e_{k}\right) \sum_{l=1}^{\min (l, \mathrm{mb}\}} s_{l} \text {. }
$$

Further, each arc $\left(E_{k}, T\right)$ has capacity $p(k, m)$. This is a special case of the polymatroidal network flow model [11], in which the capacities are defined by nonnegative and submodular functions, one for each set of arcs entering (or leaving) a specific vertez; the model derives its name from the fact that such a set function corresponds to the rank function of a polymatroid. Traditional notions such as augmenting paths and labeling techniques can be extended to find a maximum value flow for the scheduling model in $O\left(\left(m^{2} n^{3}+n^{4}\right)(m+\log n)\right)$ time $[13,14]$.

To determine the optimum value of $L_{\max }$, one again uses the concept of critical trial values so as to arrive at a polynomial-time algorithm that requires $O\left(n^{2}+n \log s_{1}+\log \left(\max \left\{d_{f}\right\}+P\right)\right)$ calls to the feasibility routine [14]. Admittedly, the degree of the polynomial is on the high side. By way of compensation, we should add that the investigation of polymatroidal network flow models was inspired by this scheduling problem and has yielded a useful generalization and unification of classical network flow theory and much of the theory of matroid optimization [11].

\section{Total Weighted Completion Time}

We finally consider the problem of minimizing the total completion time $\sum C_{j}$ or the total weighted completion time $\sum w_{j} C_{j}$.

Let us first assume that all release dates are equal. Bruno and Gonzalez [5] proposed a simple algorithm to minimize $\sum C_{j}$ on $m$ uniform machines: order the jobs according to nondecreasing execution requirements, and schedule each successive job preemptively so as to minimize its completion time. This algorithm is illustrated in Figure 5. Obviously, it requires $O(n \log n+m n)$ time and introduces at most $(m-1)\left(n-\frac{m}{2}\right)$ preemptions.

The Bruno-Gonzalez algorithm not only minimizes $\sum C_{j}$ but also $\sum_{j=1}^{l} C_{f}$ for $l=1, \ldots, n-1$. Further, it minimizes $\sum w_{j} C_{j}$ provided that the weights are agreeable, i.e., $p_{j}<p_{k}$ implies $w_{j} \geq w_{k}$ [5].

A characteristic feature of the algorithm is that at each point in time the fastest machines are working on the jobs with the shortest remaining execution requirements. One may consider a straightforward extension to the case of arbitrary release dates, in which at each subsequent release date the above rule is applied to the available jobs. 
$m=3, s_{1}=3, s_{2}=2, s_{3}=1$
$n=4, p_{1}=3, p_{2}=8, p_{3}=8, p_{4}=10$

optimal schedule obtained by Bruno-Gonzalez algorithm:

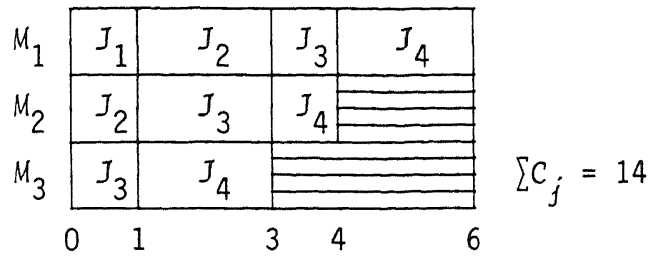

Figure 5 - Example with $m$ uniform machines, all $r_{j}=0, \sum c_{j}$ criterion.

In contrast to the algorithm described in Section 2, the resulting algorithm has the property that the remaining execution requirements passed on to the nert interval will be as unevenly distributed as possible. Unfortunately, it may produce non-optimal schedules, as is illustrated in Figure 6. The example shows in fact that no on-line algorithm will be able to minimize $\sum C_{f}$ even on two identical machines.

For the case of a single machine, it has been pointed out in Section 1 that when all release dates are equal $\sum w_{j} C_{j}$ is minimized in $O(n \log n)$ time by scheduling the jobs in order of nonincreasing ratios $w_{j} / p_{j}[19]$. Again, an obvious extension to the case of arbitrary release dates is to apply the ratio rule at each release date to the remaining execution requirements of the available jobs. This on-line algorithm yields an optimal schedule when the weights are equal or agreeable [1]. Surprisingly [1,p.82], the problem is NP-hard when the weights are arbitrary, as will be shown below.

This result will be obtained by a reduction from the following NP. complete problem [4]:

PARTITION: Given a set $T=\{1, \ldots, t\}$ and positive integers $a_{1}, \ldots, a_{i}, b$ with $\sum_{j \in T} a_{j}=2 b$, does there exist a subset $S \subset T$ such that $\sum_{j \in s} a_{j}=b$ ?

Given any instance of PARTTIION, we define a corresponding instance of the problem of minimizing $\sum w_{j} C_{j}$ on a single machine subject to arbitrary release dates as follows:

$$
\begin{aligned}
& n=t+1 ; \\
& r_{j}=0, p_{j}=w_{j}=a_{j}(j \in T) ; \\
& r_{n}=b, p_{n}=1, w_{n}=2 .
\end{aligned}
$$




$$
\begin{array}{r}
n=5, r_{1}=r_{2}=r_{3}=0, r_{4}=r_{5}=r \\
p_{1}=p_{2}=p_{3}=2, p_{4}=p_{5}=1
\end{array}
$$

(a) $r=2$

optimal schedule obtained by extended Bruno-Gonzalez algorithm:

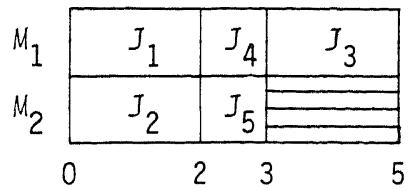

$$
\sum C_{j}=15
$$

(b) $r=3$

optimal schedule:

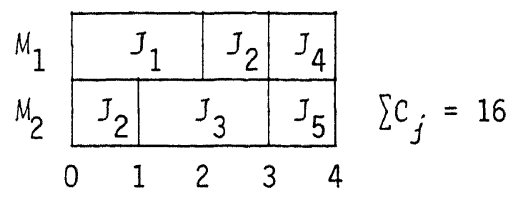

non-optimal schedule obtained by extended Bruno-Gonzalez algorithm:

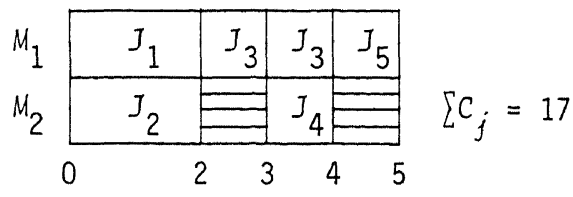

Figure 6 - Example with two identical machines, $\sum c_{j}$ criterion.

We claim that PARTITION has a solution if and only if there exists a schedule with value $\sum w, C_{j} \leq y$, where

$$
y=\sum_{1 \leq j \leq k \leq t} a_{j} a_{k}+3 b+2 \text {. }
$$

With respect to $\left\{J_{j} \mid j \in T\right\}$, any nonpreemptive schedule without machine idle time is optimal and has value $\sum_{1 \leq j \leq k \leq t} a_{j} a_{k}$. Inserting the unit-time job $J_{n}$ in a schedule for $\left\{J_{j} \mid j \in T\right\}$ increases the contribution to $\sum w_{j} C_{f}$ of the latter set by the total weight of all jobs completed after $J_{n}$. Let us denote the indez set of all jobs completed before $J_{n}$ by $S$, the length of the interval from $b$ until $J_{n}$ starts by $c(c \geq 0)$, and the length of the interval from the last completion before $J_{n}$ until $J_{n}$ starts by $d(d \geq 0)$. We then have for any schedule that

$$
\begin{gathered}
C_{n}=b+c+1, \\
\sum_{J \in S} w_{J}=b+c-d,
\end{gathered}
$$




$$
\sum w_{j} C_{j}=\sum_{1 \leq j \leq k \leq t} a_{j} a_{k}+2 b-\sum_{j \in s} w_{j}+2 C_{n}=y+c+d
$$

(cf. Figure 7). It follows that there exists a schedule with value $y$ if and only if PARTITION has a solution.

Since PARTITION can be solved in $O(t b)$ time, the above reduction does not exclude the existence of a similar pseudopolynomial algorithm [4] for the single machine problem. However, the latter problem is $N P$-hard even with respect to a unary encoding [12] (NP-hard in the strong sense [4]), which implies that it cannot be solved in pseudopolynomial time unless $P=N P$.

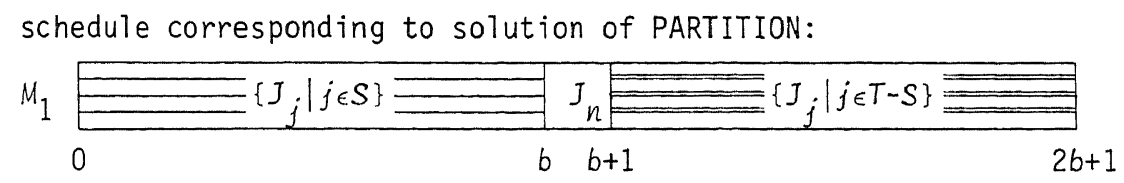

arbitrary schedule:

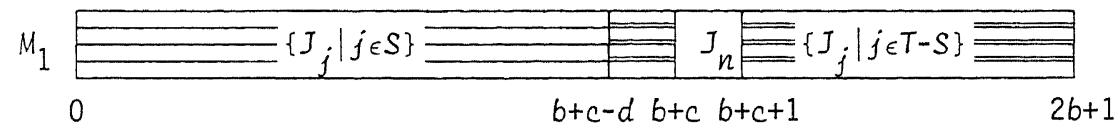

Figure 7 - Reduction from PARTITION ro single machine problem, $\sum w_{j} C_{j}$ criterion.

This stronger result can be obtained by a reduction from the following unary $N P$-complete problem [4]:

3-PARTITION: Given a set $T=\{1, \ldots, 3 t\}$ and positive integers $a_{1}, \ldots, a_{3 t}, b$ with $\frac{1}{4} b<a_{j}<\frac{1}{2} b(j \in T)$ and $\sum_{J \in T} a_{j}=t b$, do there exist $t$ pairwise disjoint subsets $s_{l} \subset T$ such that $\sum J \epsilon s_{i} a_{j}=b$ for $i=1, \ldots, t$ ?

The reduction is as follows:

$$
\begin{aligned}
& n=4 t-1 ; \\
& r_{j}=0, p_{j}=w_{j}=a_{j} \quad(j \in T) ; \\
& r_{j}=(j-3 t)(b+1)-1, p_{j}=1, w_{j}=2 \quad(j=3 t+1, \ldots, 4 t-1) ; \\
& y=\sum_{1 \leq j \leq k \leq 3 t} a_{j} a_{k}+(t-1) t\left(\frac{3}{2} b+1\right) .
\end{aligned}
$$

The equivalence proof is left to the reader. 


\section{Concluding Remarks}

The major open problem in the area of preemptive scheduling of uniform machines subject to release dates involves the minimization of $\sum C_{j}$. It has been pointed out that this problem cannot be solved by ar on-line algorithm. We suspect that it cannot be proved NP-hard either and conjecture that it is solvable in polynomial time.

An important generalization of the models considered in this paper is the addition of precedence constraints between the jobs. I1 turns out that a number of results for the nonpreemptive scheduling of unit-time jobs subject to precedence constraints can be extended to the preemptive scheduling of jobs with arbitrary processing requirements. For example, polynomial-time algorithms have been obtained for the minimization of $C_{\max }$ on an arbitrary number of identical machines subject to release dates and outtree constraints, and for the minimization of $L_{\max }$ on two uniform machines subject to release dates and general precedence constraints. Also some NP-hardness proofs carry through, e.g., for the above $C_{\max }$ problem with intree rather than outtree constraints. The reader is referred to [10] for further details.

Another challenge is to investigate the stochastic counterparts of these models, in which the job parameters are random variables and an expected objective value is to be minimized. Initial results for such models are reported in [20].

\section{Acknowledgments}

The referee's comments led to an improvemen of our running time analysis in Section 3. This research was partially supported by NSF Grants MCS76-17605 and MCS7820054 and by NATO Special Research Grant 9.2.02 (SRG. 7).

\section{References}

[1] K. R. Baker (1974) Introduction to Sequencing and Scheduling, Wiley, New York.

[2] J. Bruno, E. G. Coffman, Jr., R. Sethi (1974) Scheduling independent tasks to reduce mean finishing time. Comm. ACM 17, 382-387.

[3] J. Bruno, I. Gonzalez (1976) Schectuling independent tasks with release dates and due dates on parallel machines. Technical Report 213, Computer Science Department, Pennsylvania State University.

[4] M.R. Garey, D.S. Johnson (1979) Computers and Intractability: a Guide to the Theory of NP-Completeness, Freeman, San Francisco.

[5] I. Gonzalez (1977) Optimal mean finish time preemptive schedules. Technical Report 220, Computer Science Department, Pennsylvania State University.

[6] T. Gonzalez, S. Sahni (1978) Preemptive scheduling of uniform processor systems. J. Assoc. Comput. Mach. 25, 92-101.

[7] W.A Horn (1974) Some simple schectuling algorithms. Naval Res. Logist. Quart. $21,177-185$. 
[8] E.C. Horvath, S. Lam, R. Sechi (1977) A level algorithm for preemptive schectuling. J. Assoc. Comput. Mach. 24, 32-43.

[9] A.V. Karzanov (1974) Determining the maximal flow in a necwork by the method of preflows. Soviet Math. Dold. 15, 434437.

[10] E.I. Lawler (1982) Preemptive schectuling of precedence-constrained jobs on parallel machines. In: M. A.H. Dempster, J.K. Lenstra, A.H. G. Rinnooy Kan (eds.) (1982) Deterministic and Stochastic Scheduling, Reidel, Dordrechr, 101-123.

[11] E.I. Lawler, C.U. Martel (1982) Compuring maximal "polymatroidal" newwork flows. Math. Oper. Res. 7, 334347.

[12] J.K. Lenstra, A.H.G. Rinnooy Kan, P. Brucker (1977) Complexity of machine scheculing problems. Ann. Discrete Math. 1, 343-362.

[13] C. Martel (1982) Preemptive schectuling with release times, deadlines, and due imes. J. Assoc. Compur. Mach. 29, 812-829.

[14] C. Marrel (1982) Schectuling uniform machines with release times, deadlines and cue times. In: M.A.H. Dempster, J.K. Lenstra, A. H. G. Rinnooy Kan (eds.) (1982) Deterministic and Stochastic Schectuling, Reidel, Dordrecht, 89-99.

[15] R. McNaughton (1959) Schectuling with deadlines and loss functions. Management Sci. 6, 1-12.

[16] S. Sahni (1979) Preemptive schectuling with due dates. Oper. Res. 27, 925-934.

[17] S. Sahni, Y. Cho (1979) Nearly on line schectuling of a uniform processor system with release times. SIAM J. Comput. 8, 275-285.

[18] S. Sahni, Y. Cho (1980) Scheculing independem rasks with due times on a uniform processor system. J. Assoc. Comput. Mach. 27, 550-563.

[19] W.E. Smith (1956) Various optimizers for single-stage production. Naval Res. Logist. Quart. 3, 59-66.

[20] G. Weiss (1982) Multiserver stochastic scheduling. In: M.A.H. Dempster, J.K. Lenstra, A. H. G. Rinnooy Kan (eds.) (1982) Deterministic and Stochastic Schectuling, Reidel, Dordrecht, 157-179. 